abnormal fetal movements, multisystem neonatal disorder, and seizures and autistic features after the first year of life. Presenting features included metabolic acidosis, respiratory distress, hypotonia, food intolerance, prematurity and fetal distress. Electrolyte derangements sometimes associated may include hypocalcemia and hypomagnesaemia, and endocrine disturbances include hypothyroidism and diabetes insipidus. Neurodevelopmental outcome is impaired in the majority of cases. Diagnosis of pyridoxin dependent epilepsy is sometimes challenging because seizures are responsive to anticonvulsant drugs in $38 \%$ cases, response to pyridoxin may not be instant and obvious in $14 \%$, and structural brain abnormalities sufficient to explain the epilepsy may coexist.

Seizure types were clonic (91\%), myoclonic jerks $(62 \%)$ and tonic $(44 \%)$. EEG abnormalities included burst suppression (21\%) and hypsarrythmia (5\%). Some movement disorders were dystonic and not associated with EEG changes. MRI abnormalities inciuded cortical dysplasia, corpus callosal agenesis, and hydroccphalus, requiring v-p shunt. Biochemical and DNA tests for antiquitin deficiency and a clinical trial of pyridoxine are recommended in infants and children with epilepsy and a variety of clinical features. A clinical trial of pyridoxine should be continued for a minimum of 72 hours, with careful clinical and EEG monitoring. (Mills PB, Footitt EJ, Mills KA, et al. Genotypic and phenotypic spectrum of pyridoxine-dependent epilepsy (ALDH7A1 deficiency). Brain July 2010;133:2148-2159). (Respond: Prof PT Clayton, UCL Institute of Child Health, 30 Guilford Street, London WC1N 1EH, UK. E-Mail: p.clayton@ich.ucl.ac.uk).

COMMENT. In neonates and infants with intractable seizures, measurement of urinary alpha-aminoadipic semialdehyde and DNA tests for antiquitin deficiency should be performed to exclude pyridoxin-dependent epilepsy (PDE), regardless of an associated electrolyte disorder or structural abnormality on brain MRI. Diagnosis may be challenging because the seizures are sometimes partially controlled by anticonvulsant drugs, and the response to pyridoxine may be delayed. In classical cases of PDE, seizures occur in the first month, often within hours of birth. They are resistant to antiepileptic drugs and are controlled within an hour by $50-100 \mathrm{mg}$ pyridoxin given intravenously. A maintenance dose of $5-10 \mathrm{mg} / \mathrm{kg} /$ day of oral pyridoxin is usually sufficient to control the seizures.

\title{
DIAGNOSTIC UTILITY OF THE ICTAL CRY
}

Consecutive video-EEGs of 20 adult patients with generalized tonic-clonic (GTC) seizures and 20 with psychogenic nonepileptic seizures (PNES) were reviewed from the archives at University of South Florida, Tampa General Hospital, and the audio components of the recordings were analyzed and compared for all vocalizations. The ictal cry was defined as a prolonged tonic expiratory laryngeal vocalization, or a deep guttural clonic vocalization. The typical laryngeal sound had high sensitivity $(85 \%)$ and specificity $(100 \%)$ for epileptic GTC seizures and was not heard in any of the psychogenic cases. PNES utterances were weeping, moaning, and coughing. The ictal cry was strongly associated with epileptic GTC and warrants inquiry when taking the history from witnesses of a patient's seizure. 
In the group with epilepsy (mean age 40.2 years), 7 of 20 (41\%) patients had left temporal lobe epilepsy, 4 (23\%) right temporal lobe epilepsy, $3(11 \%)$ frontal lobe epilepsy, $2(10 \%)$ primary generalized epilepsy, and one $(5.8 \%)$ symptomatic generalized epilepsy. Three GTC events without a cry were of temporal lobe origin (2 right and 1 left). (Elzawahry H, Do CS, Lin K, Benbadis SR. The diagnostic utility of the ictal cry. Epilepsy Behav July 2010;18:306-307). (Respond: Dr H Elzawahry, 2277 Peachtree NE *302, Atlanta, GA 30309. E-mail:Hoda100@hotmail.com).

COMMENT. In a history of epilepsy in Chinese traditional medicine, cited by the authors, the ictal cry was documented by scribes (Lai C et al. Epilepsia 1991;32:229302). In centers using video-EEG, the presence or absence of an ictal cry should be of value in diagnosis of GTC vs PNES. Compared to tongue biting and urinary incontinence, having sensitivities of $23-25 \%$ for GTC (refs cited by authors), the ictal cry is of greater diagnostic value.

William Gordon Lennox, in his "Epilepsy and Related Disorders" (Boston; Little, Brown \& Co, 1960, vol 1, p186), paraphrasing Gowers (1901), recounts "the epileptic cry has been compared to the scream of a distracted peacock. Only the larynx of the bird can imitate it. A parrot in the National Hospital, London, could make the nurses come running with its scream." These descriptions aside, audio recordings of children captured in generalized tonic seizures during video-EEG might be of interest.

\section{DACHRISTIC SEIZURES WITH LEFT MESIAL TEMPORAL SCLEROSIS AND DURING WADA TEST}

Dachristic seizures are characterized by ictal crying during complex partial seizures expressed as an unconscious behavior without sadness. Researchers at the Epilepsy Monitoring Unit, Mayo Clinic, Jacksonville, FL, report a 32-year-old righthanded woman with drug resistant seizures consisting of an aura followed by staring, and crying without sadness or lacrimation. She had a history of a complex febrile seizure and status epilepticus at 10 months of age, followed by afebrile seizures in adolescence. Presurgical MRI showed left mesial temporal sclerosis. Wada testing revealed left hemisphere language dominance, and left hemisphere injection of sodium methohexital produced immediate speech arrest, right hemiplegia, and crying characteristic of the habitual seizures. Concomitant EEG revealed left hemisphere slowing. Left amygdalohippocampectomy was followed by seizure freedom for more than 2 years on tapering doses of carbamazepine. (Tatum WO, Loddenkemper T. Crying with left temporal lobe seizures and Wada testing. Epilepsy Behav July 2010;18:303-305). (Respond: Dr WO Tatum, Mayo Clinic, Jacksonville, FL 32224. E-mail: tatum.william@mayo.edu).

COMMENT. In pediatric patients, hypothalamic hamartoma and temporal lobe epilepsy are the most common localization for dachristic seizures, a rare form of epilepsy. (Luciano D et al. Crying seizures. Neurology 1993:43:2113-2117). 